\title{
Crystalline Ice: Not all bad!
}

\section{Laura Koepping, Amy Bondy and Min Su}

University of Michigan, Life Sciences Institute, Ann Arbor, Michigan, United States

Single particle sample preparation for Cryo-EM relies heavily on having the particles embedded in a thin amorphous ice layer. The most popular method to achieve this is to plunge the sample grid topped with a thin layer of sample solution into liquid ethane that is close to its freezing point $\left(-180^{\circ} \mathrm{C}\right)$ to rapidly cool down and freeze the sample solution. Even though this method is reliable in producing vitreous ice on sample grids, there are often also local areas on the grid where crystalline ice has formed. In general practice $10-20 \%$ of images in a dataset contain crystalline ice to a certain extent. Conventionally, micrographs containing these areas with crystalline ice are often sorted out of the dataset, because of concerns regarding a negative impact towards protein particles and disrupting the data processing in the $\mathrm{CTF}$ estimation and refinement steps.

In the present study we compared reconstructions from the particles extracted from micrographs containing crystalline ice and vitreous ice respectively. We further analyzed micrograph CTF fittings, ratio of well-defined particles, and image drifts for sets of micrographs with crystalline ice versus vitreous ice. We show that a moderate amount of crystalline ice in micrographs has no negative impact on the quality of the reconstruction for an Apoferritin sample dataset at sub $3 \AA$ resolution. Our findings have shown that crystalline ice could be beneficial in suppressing drift particularly during the initial few frames of the exposure.

An Apoferritin dataset was collected on a 200kV Glacios equipped with the K2 Summit direct detector at nominal pixelsize of $0.98 \AA / p x$. The following steps were performed:

- The micrographs with crystalline ice were identified manually by checking the power spectrum for the ice ring (Figure 1C). All identified micrographs formed the "crystalline ice dataset". An equalized dataset was formed from the micrographs containing no crystalline ice and named "vitreous ice dataset". A subset containing micrographs with severe crystalline ice was paired with an equalized dataset of micrographs containing no crystalline ice.

- The image shifts estimated by the motion correction were used to calculate the drift between neighboring frames. The averaged drift for each frame was plotted.

- The micrographs CTF were estimated using CTFFind4.

- Particles were picked in CryoSparc with the template picker with a low threshold set, so that all particles (even deformed or broken particles) could be picked.

- During 2D classification all classes containing Apoferritin particles were selected. For a comparison of the ratio of well-defined Apoferritin particles on micrographs with crystalline ice versus vitreous ice, all well-defined classes were selected and the ratio to total apoferritin particles was calculated (Figure 1C).

- Crystalline ice and vitreous ice datasets were regularized for defocus (Figure 2B).

- An equal number of particles from the crystalline ice datasets and the vitreous ice datasets were used in homogenous refinements. These homogenous refinements all used the same starting model derived from an ab-initio model of the combined dataset. 
All datasets led to reasonable reconstructions as compared with the published X-Ray diffraction structure. Surprisingly, we found that reconstructions of both crystalline ice datasets reached higher resolution than their comparison datasets with vitreous ice (Figure 1A). This can be visually confirmed by comparing sidechains (Figure 1B). Additionally, we looked at the ratio of Apoferritin particles in well-defined 2D-classes to total Apoferritin and used this as a measure to compare particle integrity in the different datasets. We did not find a significant difference for the ratios of particles from welldefined 2D classes for the crystalline ice dataset versus the vitreous ice dataset (Figure 1A). That suggests that the crystalline ice in our sample dataset did not seem to impact particle integrity negatively.

To explore the possible reasons for the superior resolution of the datasets with crystalline ice we compared the CTF fit resolution values as well as image drifts of the micrographs in the datasets. The average CTF fit values of the micrographs with crystalline ice were better than the micrographs containing only vitreous ice (Figure 2A), which is an indication that the micrographs in the crystalline ice datasets had on average a higher image quality. The datasets were regularized for defocus so that CTF fit resolution values would not be biased through a difference in defocus values in the two datasets (Figure 2B). We observe significantly less drift during the initial few frames for the micrographs with crystalline ice compared to the micrographs containing only vitreous ice (Figure 2C).

A similar effect was observed by Shi et all. in a preprint paper [1]. Their findings suggest that increasing vitrification temperatures (which would lead to a slower freezing of the water) suppresses the burstphase of the beam-induced motion. Formation of crystalline ice is based on slower freezing rates as well, so their ice conditions are similar to ours. The large drifts during the initial few frames often cannot be sufficiently corrected with motion correction software and so the signal cannot be recovered completely. Additionally, the signal in the initial few frames contains the highest resolution data of the undamaged particle. The suppression of larger drifts in the early frames of micrographs containing crystalline ice could also explain the improved CTF fit resolution in the crystalline ice dataset. This effect should be explored further and possibly has the potential to improve image quality lost to beam-induced motion in single-particle datasets. 
A

14969 particles from 136 micrographs with crystalline ice

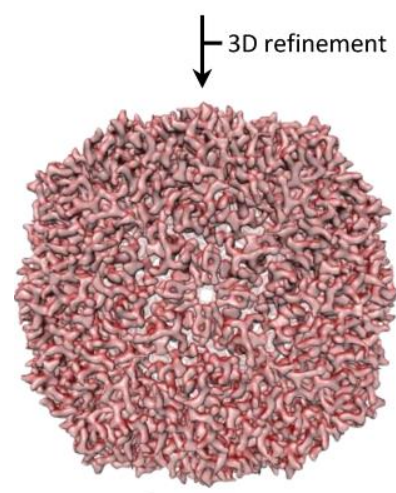

$2.7 \AA(\mathrm{FSC}=0.134)$

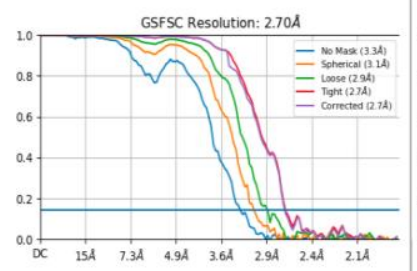

\section{B}

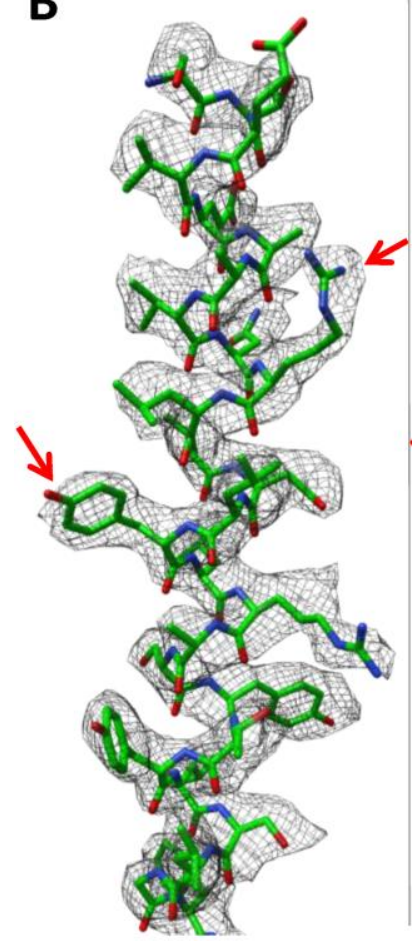

14969 particles

from 229 micrographs containing only vitreous ice

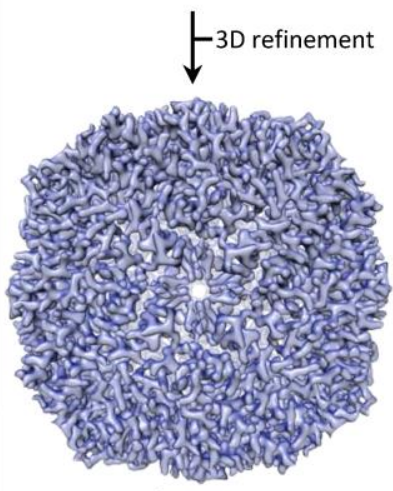

$2.84 \AA$ (FSC=0.134)
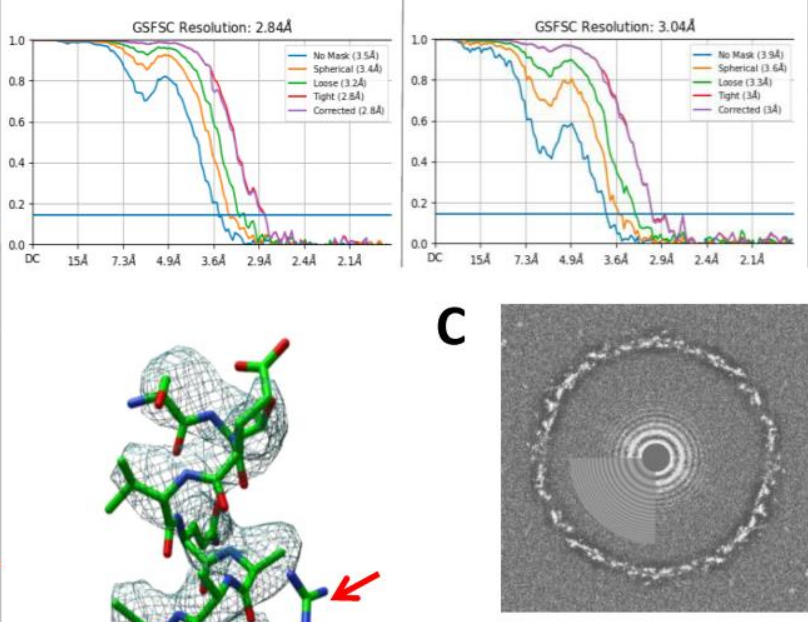

severe crystalline ice

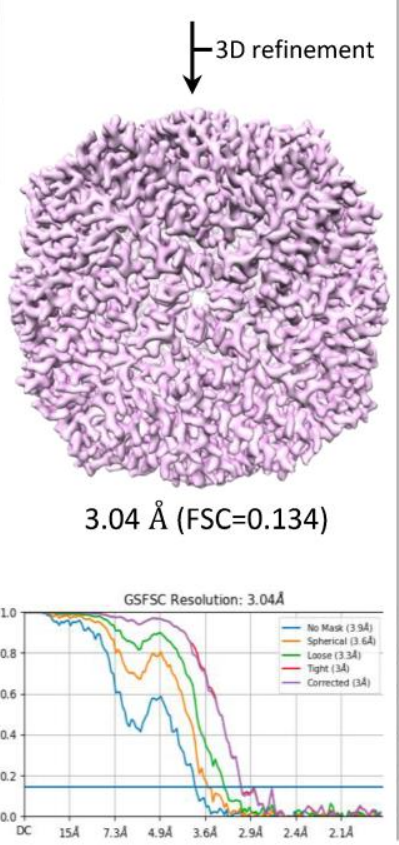

3234 particles

from 30 micrographs

containing only vitreous ice

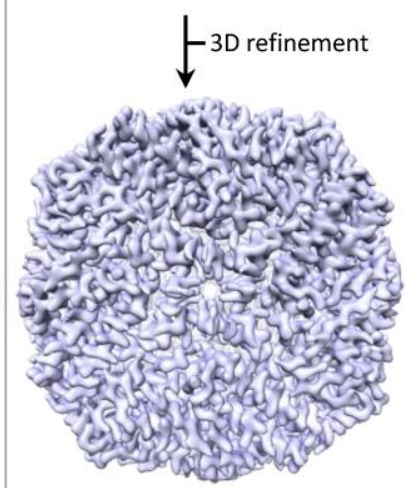

$3.34 \AA$ (FSC=0.134)
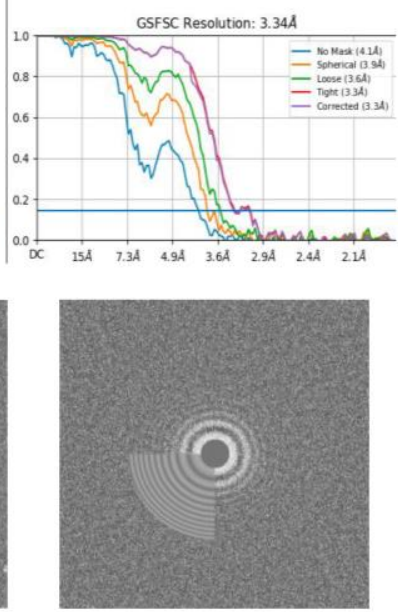

D
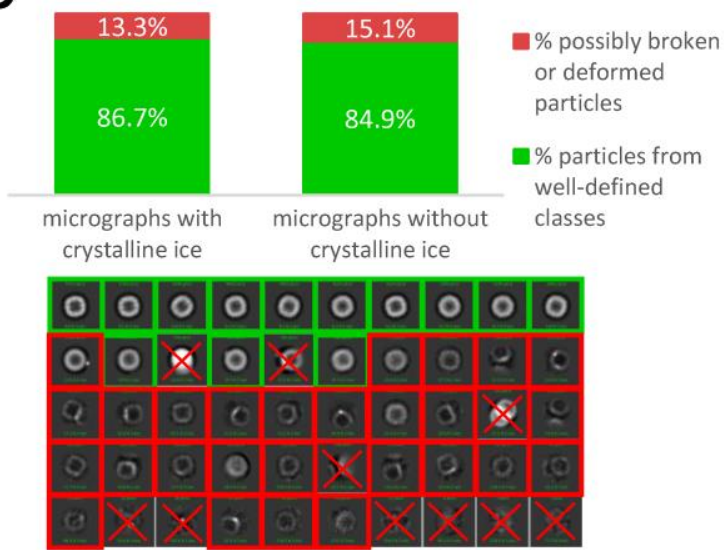

Figure 1. A 14969 particles from a dataset of 136 micrographs containing crystalline ice were refined into the reconstruction shown in red (red map; on the left) with a resolution of $2.7 \AA$. . An equalized set of particles from a dataset of micrographs containing only vitreous ice was refined into the reconstruction 
shown in blue (blue map; second from the left) with a resolution of $2.84 \AA .3211$ particles from the set of micrographs with the most severe crystalline ice were refined into the reconstruction shown in pink with a resolution of $3.04 \AA$ (pink map; second from the right). An equalized set of particles from a dataset of micrographs containing only vitreous ice were refined into the reconstruction shown in purple (purple map; on the right) with a resolution of $3.34 \AA$. B The published Xray diffraction structure was docked into the red reconstruction and a section showing the first alpha helix is shown on the left (crystalline ice dataset). On the right the same section of the structure docked into the blue reconstruction (vitreous ice dataset) is shown. The arrows show differences in the reconstructions. C The power spectrum on the right shows the signature ice ring at $\sim 3.7 \AA$ and is corresponding to a micrograph from the crystalline ice dataset used for the red reconstruction. The power spectrum on the right is corresponding to a micrograph from the vitreous ice dataset used for the blue reconstruction. D The Apoferritin particles in well-defined classes (in green boxes) make up $86.7 \%$ of the total Apoferritin particles for the crystalline ice dataset (red map). Similarly, the Apoferritin particles in well-defined classes (in green boxes) make up $84.9 \%$ of the total Apoferritin particles for the vitreous ice dataset (blue map). Red and green boxed classes combined were counted as the total Apoferritin particles. Crossed out classes (ice contamination, empty classes) were not counted in the total. 
A

CTF fit resolution of crystalline ice dataset (red map)

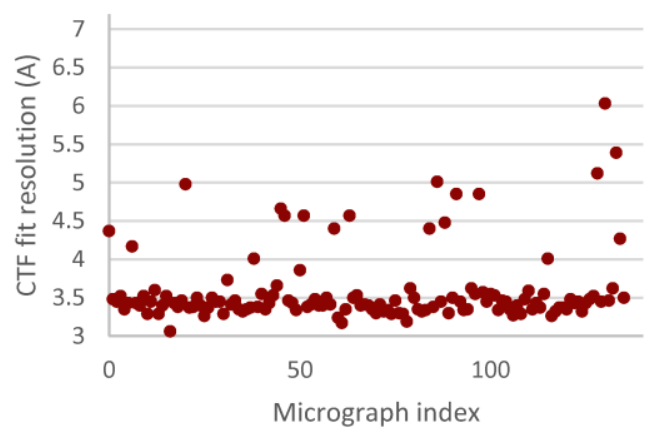

B

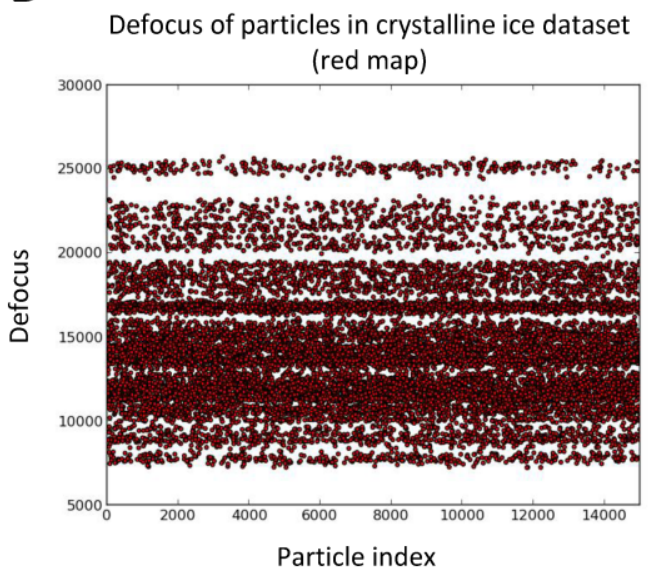

CTF fit resolution of vitreous ice dataset

(blue map)

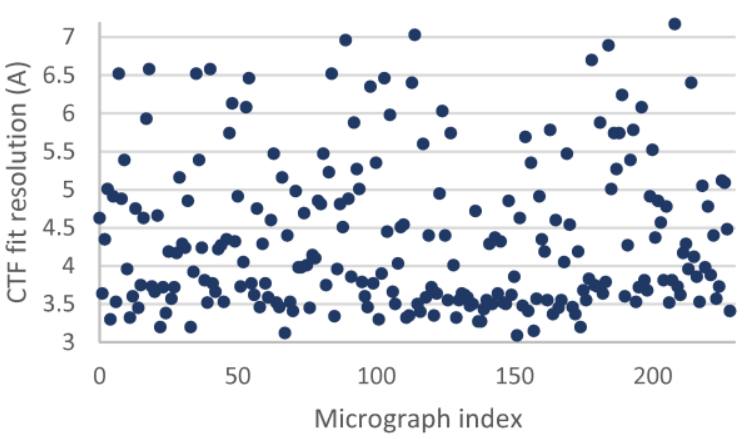

Defocus of particles in vitreous ice dataset (blue map)

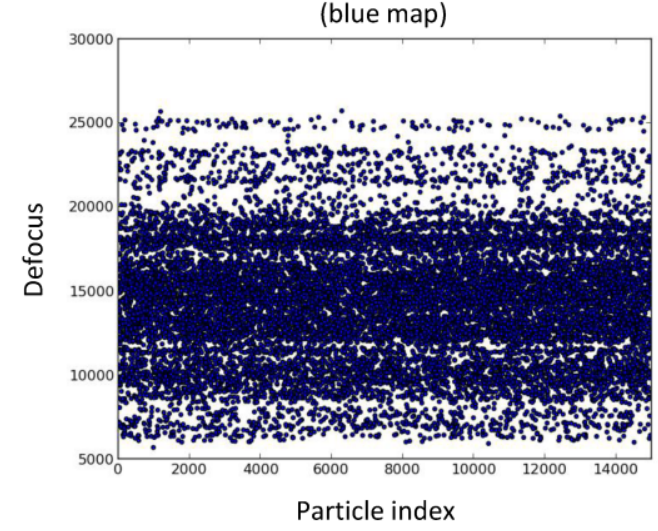

C

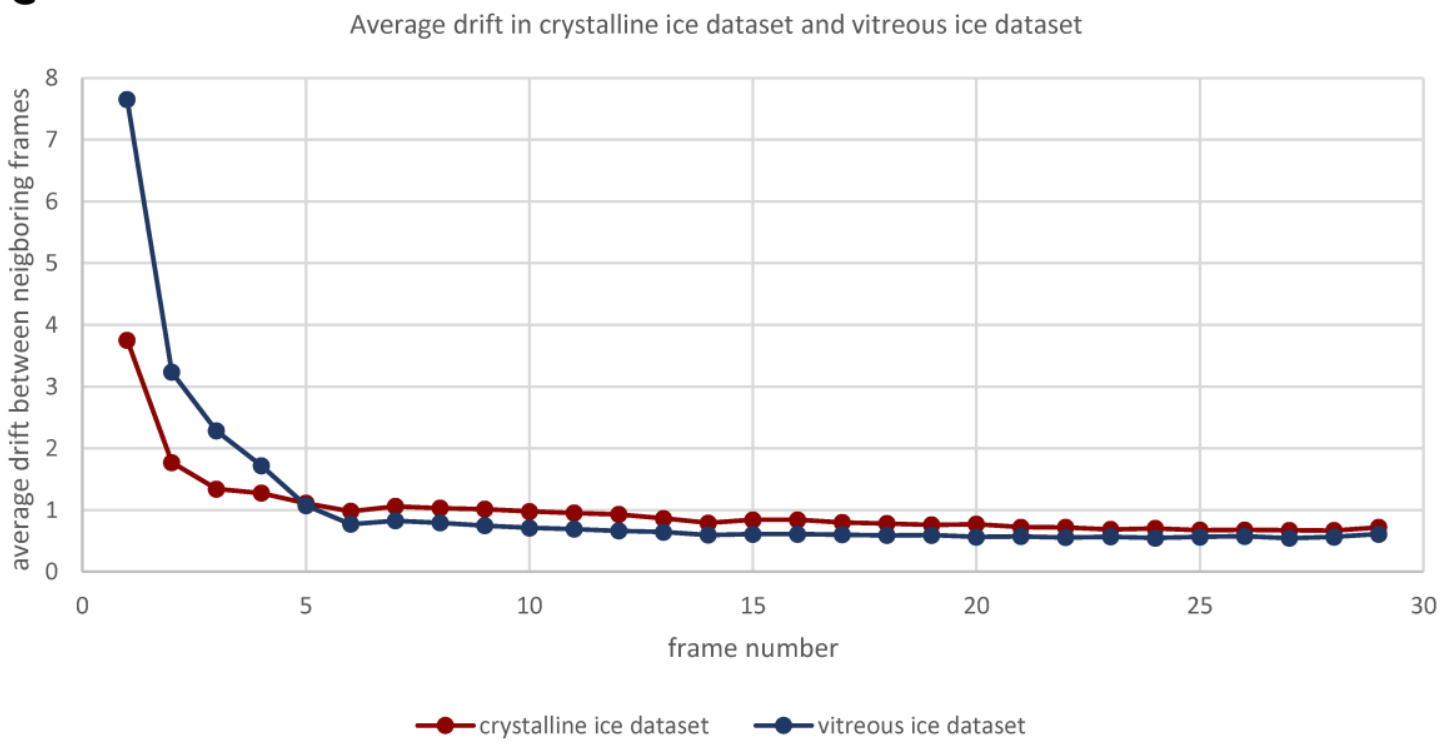

Figure 2. A CTF fit resolution values of all micrographs of the crystalline ice dataset (red map in figure 1A) are plotted on the left. The average CTF fit value is $3.59 \AA$. CTF fit resolution values of all micrographs of the vitreous ice dataset (blue map in figure 1A) are plotted on the left. The average CTF 
fit value is $4.36 \AA$. B The defocus values of all particles of the crystalline ice dataset (red map in figure 1A) are plotted on the left. The defocus values of all particles of the vitreous ice dataset (blue map in figure 1A) are plotted on the left. $\mathrm{C}$ The image drift estimated by the motion correction for each neighboring frame was averaged among all images in a dataset. The averaged drifts of the crystalline ice dataset are plotted in red and the averaged drifts of the vitreous ice dataset are plotted in blue.

\section{References}

Shi et all., Increasing vitrification temperature improves the cryo-electron microscopy reconstruction. bioRxiv 824698 (preprint); doi: https://doi.org/10.1101/824698 